\title{
Supporting Information: Structure and Plasticity of Indoleamine 2,3-Dioxygenase 1 (IDO1)
}

\author{
Ute F. Röhrig, ${ }^{*, \dagger}$ Olivier Michielin, ${ }^{*, \dagger, \ddagger}$ and Vincent Zoete ${ }^{*, \dagger, \uparrow}$ \\ $\dagger$ SIB Swiss Institute of Bioinformatics, Molecular Modeling Group, CH-1015 Lausanne, \\ Switzerland \\ $\ddagger$ Department of Oncology, University Hospital of Lausanne (CHUV), Ludwig Cancer \\ Research-Lausanne Branch, 1011 Lausanne, Switzerland \\ \Department of Oncology UNIL-CHUV, Ludwig Lausanne Branch, 1066 Epalinges, Switzerland \\ E-mail: Ute.Roehrig@sib.swiss; Olivier.Michielin@chuv.ch; Vincent.Zoete@unil.ch
}

\section{List of Tables}

S1 IDO1 X-ray structures $\ldots \ldots \ldots \ldots \ldots \ldots$

S2 IDO1 X-ray structure ligands $\ldots \ldots \ldots \ldots \ldots \ldots \ldots \ldots \ldots \ldots \ldots$

S3 VHELIBS Classification of IDO1 Ligands and Binding Sites . . . . . . . . 13

S4 IDO1 X-ray structure ligand efficiencies . . . . . . . . . . . . 15

S5 Heme-bound structures of Hydroxyamidines . . . . . . . . . . . . 17

\section{List of Figures}

S1 Co-crystallized IDO1 ligands . . . . . . . . . . . . . . . . . . . 16

S2 Additional closed JK-loop ${ }^{\mathrm{C}}$ conformations . . . . . . . . . . . . . . 18

S3 Superposition of holo and apo IDO1 inhibitors . . . . . . . . . . . . . . 19 


\begin{tabular}{|c|c|c|c|c|c|c|c|c|}
\hline ID & $\# \mathrm{Ch}$ & Form & Res. $[\AA]$ & Rfree & DPI $[\AA]$ & Ligand of interest & Mutation & Year \\
\hline $2 \mathrm{~d} 0 \mathrm{t}$ & 2 & holo & 2.30 & 0.221 & 0.33 & imidazole & & $2006^{1}$ \\
\hline $2 \mathrm{~d} 0 \mathrm{u}$ & 2 & holo & 3.40 & 0.254 & 0.96 & cyanide & & $2006^{1}$ \\
\hline $4 \mathrm{pk} 5$ & 2 & holo & 2.79 & 0.256 & 0.61 & triazothiazole & & $2014^{2}$ \\
\hline $4 \mathrm{pk} 6$ & 2 & holo & 3.45 & 0.256 & 1.01 & imidazothiazole & & $2014^{2}$ \\
\hline $4 u 72$ & 2 & holo & 2.00 & 0.231 & 0.23 & imidazole & $\mathrm{A} 260 \mathrm{G}$ & 2015 \\
\hline $4 u 74$ & 2 & holo & 2.31 & 0.220 & 0.31 & imidazole & G262A & 2015 \\
\hline $5 \mathrm{ek} 2$ & 2 & holo & 2.68 & 0.231 & 0.47 & imidazoisoindole & & $2015^{3}$ \\
\hline $5 \mathrm{ek} 3$ & 2 & holo & 2.21 & 0.232 & 0.31 & imidazoisoindole & & $2015^{3}$ \\
\hline $5 \mathrm{ek} 4$ & 2 & holo & 2.64 & 0.224 & 0.45 & imidazoisoindole & & $2015^{3}$ \\
\hline 5 etw & 2 & holo & 2.70 & 0.216 & 0.46 & pyridine & & $2016^{3}$ \\
\hline 5 whr & 2 & holo & 2.28 & 0.228 & 0.32 & indole & & $2017^{4}$ \\
\hline $5 \mathrm{wmu}$ & 2 & holo & 2.40 & 0.258 & 0.42 & cyanide/Trp & & $2017^{5}$ \\
\hline $5 \mathrm{wmv}$ & 2 & holo & 2.60 & 0.260 & 0.51 & cyanide/Trp/3ide & & $2017^{5}$ \\
\hline $5 \mathrm{wmw}$ & 2 & holo & 3.03 & 0.259 & 0.77 & cyanide/Trp & $\mathrm{F} 270 \mathrm{G}$ & $2017^{5}$ \\
\hline $5 \mathrm{wmx}$ & 2 & holo & 2.69 & 0.254 & 0.54 & cyanide/Trp/3ide & $\mathrm{F} 270 \mathrm{G}$ & $2017^{5}$ \\
\hline $5 \mathrm{wn} 8$ & 2 & holo & 2.50 & 0.252 & 0.44 & hydroxyamidine & & $2017^{5}$ \\
\hline $5 \mathrm{xe} 1$ & 2 & holo & 3.20 & 0.274 & 0.90 & hydroxyamidine & & $2017^{6}$ \\
\hline $6 \mathrm{azu}$ & 4 & holo & 2.82 & 0.272 & 0.60 & & & $2018^{7}$ \\
\hline $6 a z v$ & 4 & apo & 2.76 & 0.272 & 0.82 & phenylurea & & $2018^{7}$ \\
\hline $6 a z w$ & 2 & apo & 2.78 & 0.299 & 0.95 & N-phenyl-amide & & $2018^{7}$ \\
\hline $6 c x u$ & 2 & holo & 2.49 & 0.250 & 0.44 & cyanide/Trp & $\mathrm{S} 167 \mathrm{H}$ & $2018^{8}$ \\
\hline $6 c x v$ & 2 & holo & 2.60 & 0.266 & 0.52 & cyanide/Trp/3ide & $\mathrm{S} 167 \mathrm{H}$ & $2018^{8}$ \\
\hline 6dpq-A & 2 & holo & 2.94 & 0.240 & 0.63 & & & $2018^{9}$ \\
\hline 6dpq-B & 2 & apo & 2.94 & 0.240 & 0.63 & N-phenyl-amide & & $2018^{9}$ \\
\hline 6dpr-A & 2 & holo & 3.20 & 0.242 & 0.78 & N-phenyl-amide & & $2018^{9}$ \\
\hline 6dpr-B & 2 & apo & 3.20 & 0.242 & 0.78 & N-phenyl-amide & & $2018^{9}$ \\
\hline $6 \mathrm{e} 35$ & 2 & holo & 2.41 & 0.236 & 0.39 & cyanide/Trp/3ide & & 2018 \\
\hline $6 \mathrm{e} 40$ & 4 & holo & 2.31 & 0.254 & 0.39 & hydroxyamidine & $\mathrm{K} 116 \mathrm{~A}, \mathrm{~K} 117 \mathrm{~A}$ & $2018^{10}$ \\
\hline $6 \mathrm{e} 41$ & 4 & holo & 2.29 & 0.251 & 0.38 & hydroxyamidine & $\mathrm{K} 116 \mathrm{~A}, \mathrm{~K} 117 \mathrm{~A}$ & $2018^{10}$ \\
\hline $6 \mathrm{e} 42$ & 4 & holo & 2.10 & 0.240 & 0.30 & imidazole & $\mathrm{K} 116 \mathrm{~A}, \mathrm{~K} 117 \mathrm{~A}$ & $2018^{10}$ \\
\hline $6 \mathrm{e} 43$ & 4 & apo & 1.71 & 0.231 & 0.17 & phenylurea & $\mathrm{K} 116 \mathrm{~A}, \mathrm{~K} 117 \mathrm{~A}$ & $2018^{10}$ \\
\hline
\end{tabular}




\begin{tabular}{|c|c|c|c|c|c|c|c|c|}
\hline $6 \mathrm{e} 44$ & 4 & holo & 1.90 & 0.235 & 0.23 & & $\mathrm{~K} 116 \mathrm{~A}, \mathrm{~K} 117 \mathrm{~A}$ & $2018^{10}$ \\
\hline $6 \mathrm{e} 45$ & 4 & holo/2+ & 2.00 & 0.226 & 0.25 & & $\mathrm{~K} 116 \mathrm{~A}, \mathrm{~K} 117 \mathrm{~A}$ & $2018^{10}$ \\
\hline $6 \mathrm{e} 46$ & 4 & holo $/{ }^{2+}$ & 2.09 & 0.247 & 0.30 & $\operatorname{Trp}$ & K116A, K117A & $2018^{10}$ \\
\hline $6 \mathrm{fOa}$ & 2 & holo & 2.26 & 0.225 & 0.31 & triazole & & $2017^{11}$ \\
\hline $6 \mathrm{kof}$ & 2 & holo & 2.26 & 0.227 & 0.32 & imidazothiazole & & $2020^{12}$ \\
\hline $6 \mathrm{kps}$ & 2 & holo & 2.25 & 0.243 & 0.33 & imidazothiazole & & $2020^{12}$ \\
\hline $6 \mathrm{kw} 7$ & 2 & holo & 3.02 & 0.251 & 0.71 & imidazothiazole & & $2020^{12}$ \\
\hline 6mq6-A & 2 & holo & 3.05 & 0.229 & 0.66 & & & $2018^{9}$ \\
\hline $6 \mathrm{mq} 6-\mathrm{B}$ & 2 & apo & 3.05 & 0.229 & 0.66 & N-phenyl-amide & & $2018^{9}$ \\
\hline $6 o 3 \mathrm{i}$ & 2 & holo & 2.69 & 0.264 & 0.56 & imidazoisoindole & & $2019^{13}$ \\
\hline $6 \mathrm{pu} 7$ & 2 & holo & 2.43 & 0.250 & 0.42 & hydroxyamidine & & $2019^{14}$ \\
\hline $6 \mathrm{pz} 1$ & 2 & holo & 2.65 & 0.270 & 0.55 & indole & & $2020^{15}$ \\
\hline $6 \mathrm{r} 63$ & 2 & holo & 2.89 & 0.287 & 0.70 & triazole & & $2019^{16}$ \\
\hline 6ubp & 2 & holo & 2.95 & 0.264 & 0.69 & $\mathrm{CO} / \operatorname{Trp}$ & & $2021^{17}$ \\
\hline $6 \mathrm{v} 52$ & 2 & apo & 1.78 & 0.220 & 0.18 & benzamide & & $2020^{18}$ \\
\hline 6wjy & 2 & apo & 1.91 & 0.208 & 0.20 & N-phenyl-amide & & $2020^{19}$ \\
\hline 6wpe & 2 & apo & 2.43 & 0.235 & 0.39 & benzamide & & $2021^{20}$ \\
\hline $6 x 5 y$ & 2 & apo & 2.65 & 0.272 & 0.55 & benzamide & & $2021^{21}$ \\
\hline $7 \mathrm{a} 62$ & 4 & holo/2+ & 2.44 & 0.257 & 0.50 & & $\mathrm{~K} 116 \mathrm{~A}, \mathrm{~K} 117 \mathrm{~A}$ & $2020^{22}$ \\
\hline $7 \mathrm{ah} 4$ & 2 & holo & 2.40 & 0.249 & 0.37 & triazole & & $2021^{23}$ \\
\hline $7 \mathrm{ah} 5$ & 2 & holo & 2.90 & 0.292 & 0.72 & triazole & & $2021^{23}$ \\
\hline $7 \mathrm{ah} 6$ & 2 & holo & 3.00 & 0.276 & 0.75 & triazole & & $2021^{23}$ \\
\hline $7 \mathrm{e} 0 \mathrm{o}$ & 2 & holo & 3.34 & 0.302 & 1.05 & indazole & & $2021^{24}$ \\
\hline $7 \mathrm{e} 0 \mathrm{p}$ & 2 & holo & 2.64 & 0.266 & 0.54 & indazole & & $2021^{24}$ \\
\hline $7 \mathrm{e} 0 \mathrm{q}$ & 2 & holo & 2.46 & 0.243 & 0.41 & indazole & & $2021^{24}$ \\
\hline $7 \mathrm{e} 0 \mathrm{~s}$ & 2 & holo & 2.71 & 0.249 & 0.51 & indazole & & $2021^{24}$ \\
\hline $7 \mathrm{e} 0 \mathrm{t}$ & 2 & holo & 2.14 & 0.272 & 0.34 & indazole & & $2021^{24}$ \\
\hline $7 \mathrm{e} 0 \mathrm{u}$ & 2 & holo & 2.28 & 0.248 & 0.35 & indazole & & $2021^{24}$ \\
\hline $7 \mathrm{~m} 63$ & 2 & apo & 3.10 & 0.285 & 0.85 & N-phenyl-amide & & $2021^{25}$ \\
\hline $7 \mathrm{~m} 7 \mathrm{~d}$ & 2 & holo & 2.60 & 0.235 & 0.45 & imidazole & & $2021^{25}$ \\
\hline
\end{tabular}


Table S1: IDO1 X-ray structures available from the PDB. PBD ID (ID), number of protein chains in the structure (\#Ch), heme co-factor status (Form), resolution (Res.), R-free value (Rfree), diffraction-component precision index (DPI), type of the ligand of interest, mutations in protein sequence (Mutation), and year of structure release (Year). The oxidation state of the heme iron is ferric if not indicated otherwise $\left(\mathrm{holo}^{2+}\right)$. Structures with a high coordinate uncertainty (DPI $>0.8 \AA$ ) are marked in red. The DPI was calculated with DPICalc from Mikko J. Vainio (https://users.abo.fi/mivainio/shaep/download.php). 3ide: 3-indoleethanol. 


\begin{tabular}{|c|c|c|c|c|c|c|c|c|c|c|c|c|c|}
\hline \multirow[b]{2}{*}{$\mathrm{PDB}$} & \multirow[b]{2}{*}{ Ch. } & \multicolumn{3}{|c|}{ HPL } & \multicolumn{3}{|c|}{ FEL } & \multicolumn{3}{|c|}{ APL } & \multicolumn{3}{|c|}{ DPL } \\
\hline & & ID & EDIA & Bfact & ID & EDIA & Bfact & ID & EDIA & Bfact & ID & EDIA & Bfact \\
\hline $2 \mathrm{~d} 0 \mathrm{t}$ & A & HEM & 0.84 & 42 & PIM & 0.95 & 34 & & & & - & & \\
\hline $2 \mathrm{~d} 0 \mathrm{t}$ & B & HEM & 0.78 & 44 & PIM & 0.98 & 40 & & & & - & & \\
\hline $2 \mathrm{~d} 0 \mathrm{u}$ & $\mathrm{A}$ & HEM & 0.54 & 29 & CYN & 0.49 & 33 & - & & & - & & \\
\hline $2 \mathrm{~d} 0 \mathrm{u}$ & B & HEM & 0.47 & 30 & CYN & 0.53 & 34 & - & & & - & & \\
\hline $4 \mathrm{pk} 5$ & $\mathrm{~A}$ & HEM & 0.77 & 35 & PKJ & 0.71 & 54 & & & & - & & \\
\hline $4 \mathrm{pk} 5$ & B & HEM & 0.68 & 42 & PKJ & 0.59 & 60 & & & & - & & \\
\hline $4 \mathrm{pk} 6$ & A & HEM & 0.41 & 60 & PKL & 0.47 & 66 & & & & - & & \\
\hline 4pk6 & B & HEM & 0.56 & 67 & PKL & 0.67 & 60 & & & & - & & \\
\hline $4 u 72^{*}$ & $\mathrm{~A}$ & HEM & 1.04 & 16 & PIM & 1.05 & 13 & & & & - & & \\
\hline $4 u 72^{*}$ & B & HEM & 1.03 & 18 & PIM & 1.05 & 14 & & & & - & & \\
\hline $4 u 74^{*}$ & A & HEM & 0.97 & 20 & PIM & 0.95 & 19 & & & & - & & \\
\hline $4 u 74^{*}$ & B & HEM & 0.95 & 22 & PIM & 0.98 & 19 & & & & - & & \\
\hline $5 \mathrm{ek} 2$ & $\mathrm{~A}$ & HEM & 0.74 & 52 & $5 \mathrm{PJ}$ & 0.91 & 52 & & & & - & & \\
\hline $5 \mathrm{ek} 2$ & B & HEM & 0.73 & 52 & $5 \mathrm{PJ}$ & 0.89 & 52 & & & & - & & \\
\hline $5 \mathrm{ek} 3$ & A & HEM & 0.94 & 37 & $5 \mathrm{PK}$ & 0.97 & 39 & & & & - & & \\
\hline $5 \mathrm{ek} 3$ & B & HEM & 0.97 & 37 & $5 \mathrm{PK}$ & 1.00 & 40 & & & & - & & \\
\hline $5 \mathrm{ek} 4$ & $\mathrm{~A}$ & HEM & 0.71 & 47 & $5 \mathrm{PF}$ & 0.84 & 42 & & & & - & & \\
\hline $5 \mathrm{ek} 4$ & B & HEM & 0.73 & 47 & $5 \mathrm{PF}$ & 0.77 & 47 & & & & - & & \\
\hline 5 etw & A & HEM & 0.49 & 81 & XNL & 0.47 & 70 & & & & - & & \\
\hline 5 etw & B & HEM & 0.56 & 64 & XNL & 0.60 & 67 & & & & - & & \\
\hline 5 whr & A & HEM & 0.92 & 44 & - & & & AOJ & 0.96 & 44 & - & & \\
\hline $5 \mathrm{whr}$ & B & HEM & 0.86 & 46 & - & & & AOJ & 0.91 & 51 & - & & \\
\hline 5 wmu & A & HEM & 0.59 & 61 & CYN & 0.73 & 59 & TRP & 0.89 & 65 & - & & \\
\hline $5 \mathrm{wmu}$ & B & HEM & 0.78 & 59 & $\mathrm{CYN}$ & 0.85 & 60 & TRP & 0.95 & 60 & - & & \\
\hline $5 \mathrm{wmv}$ & A & HEM & 0.60 & 72 & CYN & 0.65 & 74 & TRP & 0.69 & 96 & $\mathrm{ZCW}$ & 0.22 & 103 \\
\hline $5 \mathrm{wmv}$ & B & HEM & 0.72 & 72 & CYN & 0.77 & 71 & TRP & 0.79 & 77 & $\mathrm{ZCW}$ & 0.47 & 90 \\
\hline $5 \mathrm{wmw}^{*}$ & A & HEM & 0.47 & 83 & CYN & 0.61 & 84 & TRP & 0.53 & 99 & TRP & 0.05 & 135 \\
\hline 5wmw* & B & HEM & 0.46 & 95 & CYN & 0.67 & 95 & TRP & 0.53 & 100 & TRP & 0.06 & 146 \\
\hline $5 w m x^{*}$ & A & HEM & 0.45 & 91 & CYN & 0.61 & 94 & TRP & 0.46 & 123 & $\mathrm{ZCW}$ & 0.48 & 83 \\
\hline $5 w m x^{*}$ & B & HEM & 0.40 & 106 & CYN & 0.37 & 107 & TRP & 0.53 & 100 & $\mathrm{ZCW}$ & 0.37 & 91 \\
\hline
\end{tabular}




\begin{tabular}{|c|c|c|c|c|c|c|c|c|c|c|c|c|c|}
\hline 5wn8 & A & HEM & 0.67 & 70 & BBJ & 0.09 & 157 & & & & - & & \\
\hline 5wn8 & B & HEM & 0.64 & 73 & BBJ & 0.45 & 125 & & & & - & & \\
\hline $5 x e 1$ & $\mathrm{~A}$ & HEM & 0.54 & 82 & IUU & 0.53 & 110 & & & & - & & \\
\hline $5 \mathrm{xe} 1$ & B & HEM & 0.54 & 77 & IUU & 0.76 & 99 & & & & - & & \\
\hline $6 \mathrm{azu}$ & A & HEM & 0.67 & 32 & ALA & 0.67 & 49 & - & & & - & & \\
\hline $6 \mathrm{azu}$ & B & HEM & 0.60 & 31 & ALA & 0.90 & 44 & - & & & - & & \\
\hline $6 \mathrm{azu}$ & $\mathrm{C}$ & HEM & 0.59 & 46 & ALA & 0.73 & 43 & - & & & - & & \\
\hline $6 \mathrm{azu}$ & $\mathrm{D}$ & HEM & 0.70 & 49 & ALA & 0.94 & 59 & - & & & - & & \\
\hline $6 \mathrm{azv}$ & A & C4V & 0.50 & 105 & & & & & & & - & & \\
\hline $6 \mathrm{azv}$ & B & C4V & 0.59 & 117 & & & & & & & - & & \\
\hline $6 \mathrm{azv}$ & $\mathrm{C}$ & $\mathrm{C} 4 \mathrm{~V}$ & 0.60 & 144 & & & & & & & - & & \\
\hline $6 \mathrm{azv}$ & $\mathrm{D}$ & $\mathrm{C} 4 \mathrm{~V}$ & 0.50 & 195 & & & & & & & - & & \\
\hline $6 \mathrm{azw}$ & $\mathrm{A}$ & C51 & 0.47 & 82 & & & & & & & & & \\
\hline $6 \mathrm{azw}$ & B & C51 & 0.42 & 123 & & & & & & & & & \\
\hline $6 \mathrm{cxu}^{*}$ & A & HEM & 0.54 & 80 & CYN & 0.84 & 73 & TRP & 0.86 & 82 & - & & \\
\hline $6 \mathrm{cxu}^{*}$ & B & HEM & 0.58 & 82 & CYN & 0.57 & 94 & TRP & 0.86 & 77 & - & & \\
\hline $6 \mathrm{cxv}^{*}$ & $\mathrm{~A}$ & HEM & 0.71 & 60 & CYN & 0.75 & 60 & TRP & 0.86 & 61 & ZCW & 0.42 & 80 \\
\hline $6 \mathrm{cxv}^{*}$ & B & HEM & 0.70 & 62 & CYN & 0.72 & 62 & TRP & 0.84 & 57 & ZCW & 0.58 & 72 \\
\hline $6 \mathrm{dpq}$ & A & HEM & 0.48 & 101 & $\mathrm{HOH}$ & 0.78 & 80 & - & & & - & & \\
\hline $6 \mathrm{dpq}$ & B & H7P & 0.60 & 133 & & & & & & & & & \\
\hline $6 \mathrm{dpr}$ & $\mathrm{A}$ & HEM & 0.40 & 82 & - & & & - & & & - & & \\
\hline $6 \mathrm{dpr}$ & B & H7P & 0.55 & 75 & & & & & & & & & \\
\hline $6 \mathrm{e} 35$ & $\mathrm{~A}$ & HEM & 0.73 & 39 & CYN & 0.65 & 31 & TRP & 0.81 & 44 & $\mathrm{ZCW}$ & 0.49 & 70 \\
\hline $6 \mathrm{e} 35$ & B & HEM & 0.87 & 38 & CYN & 0.63 & 34 & TRP & 0.90 & 40 & - & & \\
\hline $6 \mathrm{e} 40$ & $\mathrm{~A}$ & HEM & 0.71 & 57 & BBJ & 0.38 & 72 & & & & - & & \\
\hline $6 \mathrm{e} 40$ & B & HEM & 0.69 & 55 & BBJ & 0.60 & 67 & & & & - & & \\
\hline $6 \mathrm{e} 40$ & $\mathrm{C}$ & HEM & 0.70 & 63 & - & & & - & & & - & & \\
\hline $6 \mathrm{e} 40$ & $\mathrm{D}$ & HEM & 0.74 & 52 & BBJ & 0.75 & 63 & & & & - & & \\
\hline $6 \mathrm{e} 41$ & $\mathrm{~A}$ & HEM & 0.82 & 44 & HQS & 0.85 & 55 & & & & - & & \\
\hline $6 \mathrm{e} 41$ & B & HEM & 0.86 & 44 & HQS & 0.88 & 53 & & & & - & & \\
\hline $6 \mathrm{e} 41$ & $\mathrm{C}$ & HEM & 0.84 & 46 & HQS & 0.80 & 56 & & & & - & & \\
\hline $6 \mathrm{e} 41$ & $\mathrm{D}$ & HEM & 0.88 & 42 & HQS & 0.89 & 51 & & & & - & & \\
\hline $6 \mathrm{e} 42$ & A & HEM & 0.89 & 37 & HQJ & 1.00 & 34 & & & & HQJ & 0.98 & 38 \\
\hline
\end{tabular}




\begin{tabular}{|c|c|c|c|c|c|c|c|c|c|c|c|c|}
\hline $6 \mathrm{e} 42$ & B & HEM & 0.87 & 35 & HQJ & 1.01 & 31 & & & & HQJ & 1.00 \\
\hline $6 \mathrm{e} 42$ & $\mathrm{C}$ & HEM & 0.85 & 40 & HQJ & 0.98 & 36 & & & & HQJ & 0.97 \\
\hline $6 \mathrm{e} 42$ & $\mathrm{D}$ & HEM & 0.84 & 35 & HQJ & 1.01 & 33 & & & & HQJ & 0.98 \\
\hline $6 \mathrm{e} 43$ & A & HQM & 0.99 & 30 & & & & & & & BEZ & 0.94 \\
\hline $6 \mathrm{e} 43$ & B & HQM & 1.00 & 27 & & & & & & & BEZ & 0.90 \\
\hline $6 \mathrm{e} 43$ & $\mathrm{C}$ & HQM & 1.01 & 26 & & & & & & & BEZ & 0.96 \\
\hline $6 \mathrm{e} 43$ & $\mathrm{D}$ & HQM & 1.04 & 24 & & & & & & & BEZ & 1.02 \\
\hline $6 \mathrm{e} 44$ & A & HEM & 0.90 & 38 & ALA & 0.97 & 38 & - & & & - & \\
\hline $6 \mathrm{e} 44$ & B & HEM & 0.87 & 37 & ALA & 0.77 & 42 & - & & & - & \\
\hline $6 \mathrm{e} 44$ & $\mathrm{C}$ & HEM & 0.93 & 33 & ALA & 0.99 & 32 & - & & & - & \\
\hline $6 \mathrm{e} 44$ & $\mathrm{D}$ & HEM & 0.88 & 36 & ALA & 0.86 & 40 & - & & & - & \\
\hline $6 \mathrm{e} 45$ & A & HEM & 0.93 & 33 & - & & & - & & & - & \\
\hline $6 \mathrm{e} 45$ & B & HEM & 0.92 & 32 & - & & & - & & & - & \\
\hline $6 \mathrm{e} 45$ & $\mathrm{C}$ & HEM & 0.93 & 30 & - & & & - & & & - & \\
\hline $6 \mathrm{e} 45$ & $\mathrm{D}$ & HEM & 0.92 & 29 & - & & & - & & & - & \\
\hline $6 \mathrm{e} 46$ & A & HEM & 0.88 & 37 & - & & & TRP & 0.53 & 47 & - & \\
\hline $6 \mathrm{e} 46$ & B & HEM & 0.86 & 36 & - & & & TRP & 0.85 & 41 & - & \\
\hline $6 \mathrm{e} 46$ & $\mathrm{C}$ & HEM & 0.88 & 44 & - & & & TRP & 0.68 & 51 & - & \\
\hline $6 \mathrm{e} 46$ & $\mathrm{D}$ & HEM & 0.83 & 39 & - & & & TRP & 0.81 & 47 & - & \\
\hline $6 f 0 a$ & A & HEM & 0.91 & 41 & C82 & 0.90 & 51 & & & & - & \\
\hline $6 \mathrm{fOa}$ & $\mathrm{C}$ & HEM & 0.86 & 41 & C82 & 0.77 & 53 & & & & - & \\
\hline $6 \mathrm{kof}$ & A & HEM & 0.83 & 44 & DO9 & 0.90 & 44 & & & & - & \\
\hline $6 \mathrm{kof}$ & B & HEM & 0.82 & 41 & DO9 & 0.79 & 41 & & & & - & \\
\hline $6 \mathrm{kps}$ & A & HEM & 0.73 & 48 & DU6 & 0.88 & 48 & & & & - & \\
\hline $6 \mathrm{kps}$ & B & HEM & 0.83 & 44 & DU6 & 0.73 & 44 & & & & - & \\
\hline $6 \mathrm{kw} 7$ & A & HEM & 0.41 & 76 & DYC & 0.82 & 86 & & & & - & \\
\hline 6kw7 & B & HEM & 0.52 & 68 & DYC & 0.66 & 74 & & & & - & \\
\hline $6 m q 6$ & A & HEM & 0.47 & 101 & $\mathrm{HOH}$ & 0.68 & 83 & & & & - & \\
\hline $6 \mathrm{mq} 6$ & B & H7P & 0.47 & 126 & & & & & & & & \\
\hline $603 \mathrm{i}$ & A & HEM & 0.72 & 51 & LKP & 0.77 & 51 & & & & - & \\
\hline $603 \mathrm{i}$ & B & HEM & 0.68 & 57 & LKP & 0.75 & 59 & & & & - & \\
\hline $6 \mathrm{pu} 7$ & A & HEM & 0.80 & 36 & OY4 & 0.72 & 56 & & & & - & \\
\hline $6 \mathrm{pu} 7$ & B & HEM & 0.85 & 38 & OY4 & 0.75 & 59 & & & & - & \\
\hline
\end{tabular}




\begin{tabular}{|c|c|c|c|c|c|c|c|c|c|c|c|c|c|}
\hline $6 \mathrm{pz} 1$ & $\mathrm{~A}$ & HEM & 0.77 & 75 & - & & & AOJ & 0.57 & 111 & - & & \\
\hline 6pz1 & B & HEM & 0.64 & 77 & - & & & AOJ & 0.79 & 72 & AOJ & 0.12 & 132 \\
\hline $6 \mathrm{r} 63$ & A & HEM & 0.44 & 60 & JTB & 0.67 & 59 & & & & - & & \\
\hline $6 \mathrm{r} 63$ & B & HEM & 0.37 & 66 & JTB & 0.72 & 62 & & & & JTB & 0.39 & 65 \\
\hline 6ubp & $\mathrm{A}$ & HEM & 0.42 & 98 & - & & & - & & & - & & \\
\hline 6ubp & B & HEM & 0.41 & 98 & - & & & - & & & - & & \\
\hline 6v52 & A & QPV & 1.02 & 22 & & & & & & & & & \\
\hline $6 \mathrm{v} 52$ & B & QPV & 1.02 & 24 & & & & & & & & & \\
\hline 6wjy & A & $\mathrm{U} 41$ & 1.05 & 33 & & & & & & & & & \\
\hline 6wjy & B & U41 & 1.05 & 33 & & & & & & & & & \\
\hline 6wpe & A & U6G & 0.95 & 46 & & & & & & & & & \\
\hline 6wpe & B & U6G & 0.96 & 46 & & & & & & & & & \\
\hline $6 x 5 y$ & $\mathrm{~A}$ & URJ & 0.72 & 69 & & & & & & & & & \\
\hline $6 x 5 y$ & B & URJ & 0.78 & 61 & & & & & & & & & \\
\hline 7a62 & A & HEM & 0.77 & 39 & - & & & - & & & - & & \\
\hline $7 \mathrm{a} 62$ & B & HEM & 0.75 & 43 & - & & & - & & & - & & \\
\hline $7 a 62$ & $\mathrm{C}$ & HEM & 0.62 & 50 & - & & & - & & & - & & \\
\hline 7a62 & $\mathrm{D}$ & HEM & 0.64 & 51 & - & & & - & & & - & & \\
\hline 7ah4 & A & HEM & 0.82 & 50 & $\mathrm{RCN}$ & 0.89 & 45 & & & & $\mathrm{RCN}$ & 0.80 & 48 \\
\hline 7ah4 & B & HEM & 0.74 & 45 & $\mathrm{RCN}$ & 0.97 & 42 & & & & $\mathrm{RCN}$ & 0.83 & 45 \\
\hline 7ah5 & A & HEM & 0.39 & 60 & $\mathrm{RCQ}$ & 0.79 & 53 & & & & $\mathrm{RCQ}$ & 0.74 & 61 \\
\hline 7ah5 & B & HEM & 0.39 & 54 & $\mathrm{RCQ}$ & 0.85 & 53 & & & & $\mathrm{RCQ}$ & 0.54 & 54 \\
\hline 7ah6 & A & HEM & 0.49 & 49 & $\mathrm{RCW}$ & 0.80 & 47 & & & & $\mathrm{RCW}$ & 0.75 & 45 \\
\hline 7ah6 & B & HEM & 0.50 & 62 & $\mathrm{RCW}$ & 0.91 & 55 & & & & $\mathrm{RCW}$ & 0.70 & 65 \\
\hline 7e0o & A & HEM & 0.21 & 92 & HS0 & 0.89 & 105 & & & & - & & \\
\hline $7 \mathrm{e} 0 \mathrm{o}$ & B & HEM & 0.28 & 94 & HS0 & 0.86 & 116 & & & & - & & \\
\hline $7 \mathrm{e} 0 \mathrm{p}$ & A & HEM & 0.69 & 52 & HU0 & 0.64 & 64 & & & & - & & \\
\hline $7 \mathrm{e} 0 \mathrm{p}$ & B & HEM & 0.44 & 73 & HU0 & 0.75 & 76 & & & & - & & \\
\hline $7 \mathrm{e} 0 \mathrm{q}$ & A & HEM & 0.82 & 43 & HU3 & 0.85 & 49 & & & & - & & \\
\hline $7 \mathrm{e} 0 \mathrm{q}$ & B & HEM & 0.69 & 48 & HU3 & 0.75 & 55 & & & & - & & \\
\hline $7 \mathrm{e} 0 \mathrm{~s}$ & A & HEM & 0.56 & 53 & HU6 & 0.86 & 53 & & & & - & & \\
\hline $7 \mathrm{e} 0 \mathrm{~s}$ & B & HEM & 0.64 & 50 & HU6 & 0.79 & 60 & & & & - & & \\
\hline $7 \mathrm{e} 0 \mathrm{t}$ & A & HEM & 0.88 & 53 & HU9 & 0.94 & 57 & & & & - & & \\
\hline
\end{tabular}




\begin{tabular}{ll|lll|lll|}
$7 \mathrm{e} 0 \mathrm{t}$ & B & HEM & 0.84 & 52 & HU9 & 0.88 & 57 \\
$7 \mathrm{e} 0 \mathrm{u}$ & A & HEM & 0.92 & 41 & HUC & 0.99 & 46 \\
$7 \mathrm{e} 0 \mathrm{u}$ & B & HEM & 0.70 & 50 & HUC & 0.97 & 54 \\
$7 \mathrm{~m} 63$ & A & YRP & 0.56 & 61 & & & \\
$7 \mathrm{~m} 63$ & B & YRP & 0.60 & 55 & & & \\
$7 \mathrm{~m} 7 \mathrm{~d}$ & A & HEM & 0.51 & 52 & YRM & 0.86 & 58 \\
$7 \mathrm{~m} 7 \mathrm{~d}$ & B & HEM & 0.60 & 60 & YRM & 0.95 & 64
\end{tabular}

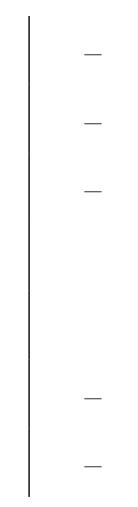

Table S2: IDO1 X-ray structure ligands. PDB ID, chain ID (Ch.), heme pocket ligand (HPL), Fe-bound ligand (FEL), A-pocket ligand (APL), D-pocket ligand (DPL). For each ligand, its PDB ID (ID), EDIAm value (EDIA) and average B-factor (Bfact) are given. Structures with active site mutations marked with an asterisk. A dash indicates an empty or closed pocket without ligand, while an empty case indicates that the last ligand to the left also fills the respective pocket. The chemical structures of the ligands with their PDB codes are given in Figure S1. 


\begin{tabular}{|c|c|c|c|c|c|c|c|c|c|c|}
\hline \multirow[b]{2}{*}{ PDB } & \multirow[b]{2}{*}{ Ch. } & \multicolumn{3}{|c|}{ FEL } & \multicolumn{3}{|c|}{ APL } & \multicolumn{3}{|c|}{ DPL } \\
\hline & & ID & Ligand & BSite & ID & Ligand & BSite & ID & Ligand & BSite \\
\hline $2 \mathrm{~d} 0 \mathrm{t}$ & $\mathrm{A}$ & PIM & Good & Dubious & & & & - & & \\
\hline $2 \mathrm{~d} 0 \mathrm{t}$ & B & PIM & Good & Dubious & & & & - & & \\
\hline $4 \mathrm{pk} 5$ & $\mathrm{~A}$ & PKJ & Good & Dubious & & & & - & & \\
\hline $4 \mathrm{pk} 5$ & B & PKJ & Good & Good & & & & - & & \\
\hline $4 \mathrm{pk} 6$ & $\mathrm{~A}$ & PKL & Dubious & Bad & & & & - & & \\
\hline $4 \mathrm{pk} 6$ & $\mathrm{~B}$ & PKL & Dubious & Dubious & & & & - & & \\
\hline $4 u 72^{*}$ & $\mathrm{~A}$ & PIM & Good & Good & & & & - & & \\
\hline $4 u 72^{*}$ & B & PIM & Good & Good & & & & - & & \\
\hline $4 u 74^{*}$ & $\mathrm{~A}$ & PIM & Good & Good & & & & - & & \\
\hline $4 u 74^{*}$ & B & PIM & Good & Good & & & & - & & \\
\hline $5 \mathrm{ek} 2$ & $\mathrm{~A}$ & $5 \mathrm{PJ}$ & Good & Dubious & & & & - & & \\
\hline $5 \mathrm{ek} 2$ & B & $5 \mathrm{PJ}$ & Dubious & Dubious & & & & - & & \\
\hline $5 \mathrm{ek} 3$ & A & $5 \mathrm{PK}$ & Good & Good & & & & - & & \\
\hline $5 \mathrm{ek} 3$ & B & $5 \mathrm{PK}$ & Good & Good & & & & - & & \\
\hline $5 \mathrm{ek} 4$ & $\mathrm{~A}$ & $5 \mathrm{PF}$ & Good & Dubious & & & & - & & \\
\hline $5 \mathrm{ek} 4$ & B & $5 \mathrm{PF}$ & Good & Good & & & & - & & \\
\hline 5etw & A & XNL & Dubious & Dubious & & & & - & & \\
\hline 5 etw & B & XNL & Dubious & Dubious & & & & - & & \\
\hline 5 whr & A & - & & & AOJ & Good & Good & - & & \\
\hline 5 whr & B & - & & & AOJ & Good & Good & - & & \\
\hline $5 \mathrm{wmu}$ & A & $\mathrm{CYN}$ & & & TRP & Dubious & Dubious & - & & \\
\hline $5 \mathrm{wmu}$ & B & CYN & & & TRP & Good & Dubious & - & & \\
\hline $5 \mathrm{wmv}$ & A & $\mathrm{CYN}$ & & & TRP & Dubious & Dubious & $\mathrm{ZCW}$ & $\mathrm{Bad}$ & Dubious \\
\hline $5 \mathrm{wmv}$ & B & $\mathrm{CYN}$ & & & TRP & Good & Dubious & $\mathrm{ZCW}$ & Dubious & Dubious \\
\hline $5 \mathrm{wmw}^{*}$ & A & $\mathrm{CYN}$ & & & TRP & Good & Dubious & TRP & Bad & Dubious \\
\hline $5 \mathrm{wmw}^{*}$ & B & $\mathrm{CYN}$ & & & TRP & Good & Dubious & TRP & Bad & Dubious \\
\hline $5 \mathrm{wmx} *$ & A & CYN & & & TRP & Dubious & Dubious & $\mathrm{ZCW}$ & Dubious & Dubious \\
\hline $5 \mathrm{wmx} *$ & B & $\mathrm{CYN}$ & & & TRP & Good & Dubious & $\mathrm{ZCW}$ & Dubious & Dubious \\
\hline 5wn8 & A & BBJ & $\mathrm{Bad}$ & Dubious & & & & - & & \\
\hline 5wn8 & B & BBJ & Dubious & Dubious & & & & - & & \\
\hline
\end{tabular}




\begin{tabular}{|c|c|c|c|c|c|c|c|c|c|c|}
\hline 5 xe1 & $\mathrm{A}$ & IUU & Dubious & Dubious & & & & - & & \\
\hline $5 x e 1$ & $\mathrm{~B}$ & IUU & Dubious & Dubious & & & & - & & \\
\hline $6 \mathrm{azv}$ & $\mathrm{A}$ & - & & & $\mathrm{C} 4 \mathrm{~V}$ & Dubious & Dubious & - & & \\
\hline $6 \mathrm{azv}$ & $\mathrm{B}$ & - & & & $\mathrm{C} 4 \mathrm{~V}$ & Dubious & Dubious & - & & \\
\hline $6 \mathrm{azv}$ & $\mathrm{C}$ & - & & & $\mathrm{C} 4 \mathrm{~V}$ & Dubious & Dubious & - & & \\
\hline $6 a z v$ & $\mathrm{D}$ & - & & & $\mathrm{C} 4 \mathrm{~V}$ & Dubious & Dubious & - & & \\
\hline $6 \mathrm{azw}$ & $\mathrm{A}$ & - & & & C51 & Good & Bad & & & \\
\hline $6 \mathrm{azw}$ & $\mathrm{B}$ & - & & & C51 & Dubious & Dubious & & & \\
\hline $6 \mathrm{cxu}^{*}$ & $\mathrm{~A}$ & CYN & & & TRP & Dubious & Dubious & - & & \\
\hline $6 \mathrm{cxu}^{*}$ & $\mathrm{~B}$ & $\mathrm{CYN}$ & & & TRP & Dubious & Dubious & - & & \\
\hline $6 \mathrm{cxv}^{*}$ & $\mathrm{~A}$ & $\mathrm{CYN}$ & & & TRP & $\mathrm{Bad}$ & Bad & ZCW & $\mathrm{Bad}$ & Bad \\
\hline $6 \mathrm{cxv}^{*}$ & $\mathrm{~B}$ & $\mathrm{CYN}$ & & & TRP & $\mathrm{Bad}$ & Bad & ZCW & Bad & Bad \\
\hline $6 \mathrm{dpq}$ & B & - & & & $\mathrm{H} 7 \mathrm{P}$ & Dubious & Dubious & & & \\
\hline $6 \mathrm{dpr}$ & $\mathrm{B}$ & - & & & $\mathrm{H} 7 \mathrm{P}$ & Good & Dubious & & & \\
\hline $6 \mathrm{e} 35$ & $\mathrm{~A}$ & CYN & & & TRP & Good & Good & ZCW & Good & Good \\
\hline $6 \mathrm{e} 35$ & $\mathrm{~B}$ & $\mathrm{CYN}$ & & & TRP & Good & Good & - & & \\
\hline $6 \mathrm{e} 40$ & $\mathrm{~A}$ & BBJ & Dubious & Dubious & & & & - & & \\
\hline $6 \mathrm{e} 40$ & $\mathrm{~B}$ & BBJ & Good & Good & & & & - & & \\
\hline $6 \mathrm{e} 40$ & $\mathrm{D}$ & BBJ & Good & Dubious & & & & - & & \\
\hline $6 \mathrm{e} 41$ & $\mathrm{~A}$ & HQS & Good & Good & & & & - & & \\
\hline $6 \mathrm{e} 41$ & B & HQS & Good & Dubious & & & & - & & \\
\hline $6 \mathrm{e} 41$ & $\mathrm{C}$ & HQS & Good & Dubious & & & & - & & \\
\hline $6 \mathrm{e} 41$ & $\mathrm{D}$ & HQS & Good & Dubious & & & & - & & \\
\hline $6 \mathrm{e} 42$ & $\mathrm{~A}$ & HQJ & Good & Good & & & & HQJ & Good & Good \\
\hline $6 \mathrm{e} 42$ & $\mathrm{~B}$ & HQJ & Good & Good & & & & HQJ & Good & Good \\
\hline $6 \mathrm{e} 42$ & $\mathrm{C}$ & HQJ & Good & Good & & & & HQJ & Good & Good \\
\hline $6 \mathrm{e} 42$ & $\mathrm{D}$ & HQJ & Good & Good & & & & HQJ & Good & Good \\
\hline $6 \mathrm{e} 43$ & $\mathrm{~A}$ & - & & & HQM & Good & Good & BEZ & & \\
\hline $6 \mathrm{e} 43$ & $\mathrm{~B}$ & - & & & HQM & Good & Good & BEZ & & \\
\hline $6 \mathrm{e} 43$ & $\mathrm{C}$ & - & & & HQM & Good & Good & BEZ & & \\
\hline $6 \mathrm{e} 43$ & $\mathrm{D}$ & - & & & HQM & Good & Good & BEZ & & \\
\hline 6f0a & A & C82 & Good & Good & & & & - & & \\
\hline $6 \mathrm{f0a}$ & $\mathrm{C}$ & $\mathrm{C} 82$ & Good & Good & & & & - & & \\
\hline
\end{tabular}




\begin{tabular}{|c|c|c|c|c|c|c|c|c|c|c|}
\hline 6kof & $\mathrm{A}$ & DO9 & Good & Dubious & & & & - & & \\
\hline $6 \mathrm{kof}$ & $\mathrm{B}$ & DO9 & Good & Dubious & & & & - & & \\
\hline 6kps & $\mathrm{A}$ & DU6 & Good & Dubious & & & & - & & \\
\hline 6kps & $\mathrm{B}$ & DU6 & Good & Dubious & & & & - & & \\
\hline $6 \mathrm{kw} 7$ & $\mathrm{~A}$ & DYC & Dubious & Bad & & & & - & & \\
\hline 6kw7 & $\mathrm{B}$ & DYC & Good & Dubious & & & & - & & \\
\hline $6 \mathrm{mq} 6$ & $\mathrm{~B}$ & - & & & $\mathrm{H} 7 \mathrm{P}$ & Dubious & Bad & & & \\
\hline $603 \mathrm{i}$ & $\mathrm{A}$ & LKP & Good & Dubious & & & & - & & \\
\hline $603 \mathrm{i}$ & $\mathrm{B}$ & LKP & Good & Dubious & & & & - & & \\
\hline 6pu7 & $\mathrm{A}$ & OY4 & Good & Good & & & & - & & \\
\hline 6pu7 & $\mathrm{B}$ & OY4 & Good & Good & & & & - & & \\
\hline $6 \mathrm{pz} 1$ & $\mathrm{~A}$ & - & & & AOJ & Dubious & Dubious & - & & \\
\hline $6 \mathrm{pz} 1$ & B & - & & & AOJ & Good & Dubious & AOJ & Bad & Dubious \\
\hline $6 \mathrm{r} 63$ & $\mathrm{~A}$ & JTB & Dubious & Dubious & & & & - & & \\
\hline $6 \mathrm{r} 63$ & B & JTB & Bad & Dubious & & & & JTB & Bad & Bad \\
\hline 6ubp & $\mathrm{A}$ & - & & & - & & & - & & \\
\hline 6ubp & $\mathrm{B}$ & - & & & - & & & - & & \\
\hline $6 \mathrm{v} 52$ & $\mathrm{~A}$ & - & & & QPV & Good & Good & & & \\
\hline $6 \mathrm{v} 52$ & $\mathrm{~B}$ & - & & & QPV & Good & Good & & & \\
\hline 6wjy & $\mathrm{A}$ & - & & & U41 & Good & Dubious & & & \\
\hline 6wjy & B & - & & & $\mathrm{U} 41$ & Good & Good & & & \\
\hline 6wpe & $\mathrm{A}$ & - & & & U6G & Good & Good & & & \\
\hline 6wpe & $\mathrm{B}$ & - & & & $\mathrm{U} 6 \mathrm{G}$ & Good & Good & & & \\
\hline $6 x 5 y$ & $\mathrm{~A}$ & - & & & URJ & Good & Dubious & & & \\
\hline $6 x 5 y$ & B & - & & & URJ & Good & Dubious & & & \\
\hline $7 \mathrm{ah} 4$ & $\mathrm{~A}$ & $\mathrm{RCN}$ & Dubious & Dubious & & & & $\mathrm{RCN}$ & Dubious & Dubious \\
\hline $7 \mathrm{ah} 4$ & $\mathrm{~B}$ & $\mathrm{RCN}$ & Dubious & Dubious & & & & $\mathrm{RCN}$ & Dubious & Dubious \\
\hline $7 \mathrm{ah} 5$ & $\mathrm{~A}$ & $\mathrm{RCQ}$ & Dubious & Dubious & & & & $\mathrm{RCQ}$ & Dubious & Dubious \\
\hline $7 \mathrm{ah} 5$ & $\mathrm{~B}$ & $\mathrm{RCQ}$ & Dubious & Dubious & & & & $\mathrm{RCQ}$ & Bad & Dubious \\
\hline 7ah6 & $\mathrm{A}$ & $\mathrm{RCW}$ & Dubious & Dubious & & & & $\mathrm{RCW}$ & Dubious & Dubious \\
\hline 7ah6 & B & $\mathrm{RCW}$ & Dubious & Dubious & & & & RCW & Bad & Dubious \\
\hline $7 \mathrm{e} 0 \mathrm{o}$ & $\mathrm{A}$ & HS0 & Good & Dubious & & & & - & & \\
\hline $7 \mathrm{e} 0 \mathrm{o}$ & $\mathrm{B}$ & HS0 & Dubious & Dubious & & & & - & & \\
\hline
\end{tabular}




\begin{tabular}{|c|c|c|c|c|c|c|c|}
\hline $7 \mathrm{e} 0 \mathrm{p}$ & A & HU0 & Good & Dubious & & & \\
\hline $7 \mathrm{e} 0 \mathrm{p}$ & B & HU0 & Good & Dubious & & & \\
\hline $7 \mathrm{e} 0 \mathrm{q}$ & A & HU3 & Good & Good & & & \\
\hline $7 \mathrm{e} 0 \mathrm{q}$ & B & HU3 & Good & Good & & & \\
\hline $7 \mathrm{e} 0 \mathrm{~s}$ & A & HU6 & Good & Dubious & & & \\
\hline $7 \mathrm{e} 0 \mathrm{~s}$ & B & HU6 & Good & Dubious & & & \\
\hline $7 \mathrm{e} 0 \mathrm{t}$ & A & HU9 & Good & Dubious & & & \\
\hline $7 \mathrm{e} 0 \mathrm{t}$ & B & HU9 & Good & Dubious & & & \\
\hline $7 \mathrm{e} 0 \mathrm{u}$ & A & HUC & Good & Dubious & & & \\
\hline $7 \mathrm{e} 0 \mathrm{u}$ & B & HUC & Good & Dubious & & & \\
\hline $7 \mathrm{~m} 63$ & A & - & & & YRP & Dubious & Dubious \\
\hline $7 \mathrm{~m} 63$ & B & - & & & YRP & Dubious & Dubious \\
\hline $7 \mathrm{~m} 7 \mathrm{~d}$ & A & YRM & Good & Dubious & & & \\
\hline $7 \mathrm{~m} 7 \mathrm{~d}$ & B & YRM & Good & Dubious & & & \\
\hline
\end{tabular}

Table S3: VHELIBS classification of IDO1 ligands and binding sites (BSite). Ligands without classification (CYN, BEZ) are not recognized as ligands by the algorithm. VHELIBS 5.1. was used for analysis. ${ }^{26}$ 


\begin{tabular}{|c|c|c|c|c|c|}
\hline PDB ID & $\mathrm{IC}_{50}[\mu \mathrm{M}]$ & Act $[\mathrm{kcal} / \mathrm{mol}]$ & HAtoms & $\mathrm{LE}[\mathrm{kcal} / \mathrm{mol}]$ & Pockets \\
\hline $5 \mathrm{PF}$ & 0.019 & -10.52 & 22 & -0.48 & $A+B$ \\
\hline $5 \mathrm{PJ}$ & 0.280 & -8.93 & 22 & -0.41 & $A+B$ \\
\hline $5 \mathrm{PK}$ & 0.038 & -10.11 & 21 & -0.48 & $\mathrm{~A}+\mathrm{B}$ \\
\hline AOJ & 0.410 & -8.70 & 17 & -0.51 & $A+B$ \\
\hline BBJ & 0.073 & -9.73 & 25 & -0.39 & $A+B$ \\
\hline $\mathrm{C} 82$ & 11.300 & -6.74 & 13 & -0.52 & $\mathrm{~A}$ \\
\hline DO9 & 0.016 & -10.62 & 26 & -0.41 & $\mathrm{~A}+\mathrm{B}$ \\
\hline DU6 & 0.100 & -9.54 & 26 & -0.37 & $\mathrm{~A}+\mathrm{B}$ \\
\hline DYC & 0.380 & -8.75 & 15 & -0.58 & $\mathrm{~A}$ \\
\hline HQJ & 2.600 & -7.61 & 12 & -0.63 & $\mathrm{~A}$ \\
\hline HSO & 9.500 & -6.84 & 11 & -0.62 & $\mathrm{~A}$ \\
\hline HU0 & 5.600 & -7.16 & 19 & -0.38 & $A+B$ \\
\hline HU3 & 2.900 & -7.55 & 19 & -0.40 & $A+B$ \\
\hline HU6 & 0.640 & -8.44 & 19 & -0.44 & $A+B$ \\
\hline HU9 & 1.230 & -8.05 & 19 & -0.42 & $\mathrm{~A}+\mathrm{B}$ \\
\hline HUC & 0.290 & -8.91 & 19 & -0.47 & $\mathrm{~A}+\mathrm{B}$ \\
\hline IUU & 0.067 & -9.78 & 18 & -0.54 & $A+B$ \\
\hline JTB & 0.059 & -9.85 & 13 & -0.76 & $\mathrm{~A}$ \\
\hline LKP & 0.028 & -10.29 & 23 & -0.45 & $A+B$ \\
\hline OY4 & 0.035 & -10.16 & 25 & -0.41 & $\mathrm{~A}+\mathrm{B}$ \\
\hline PIM & 48.000 & -5.89 & 11 & -0.54 & $\mathrm{~A}$ \\
\hline PKJ & 3.000 & -7.53 & 29 & -0.26 & $\mathrm{~A}+\mathrm{B}$ \\
\hline PKL & 1.900 & -7.80 & 27 & -0.29 & $\mathrm{~A}+\mathrm{B}$ \\
\hline $\mathrm{RCN}$ & 1.500 & -7.94 & 13 & -0.61 & $\mathrm{~A}$ \\
\hline $\mathrm{RCQ}$ & 0.035 & -10.16 & 13 & -0.78 & $\mathrm{~A}$ \\
\hline $\mathrm{RCW}$ & 0.024 & -10.38 & 13 & -0.80 & $\mathrm{~A}$ \\
\hline
\end{tabular}




\begin{tabular}{l|lll|l} 
YRM & 0.024 & -10.38 & 26 & -0.40
\end{tabular}

Table S4: Enzymatic efficiencies of co-crystallized holo-IDO1 inhibitors. Activity (Act = $-R T \log \left(I C_{50}\right)$ ), number of heavy atoms (HAtoms), ligand efficiency (LE = Act/HAtoms), active site pockets occupied by the ligand (Pockets). The chemical structures of the ligands with their PDB codes are given in Figure S1. 

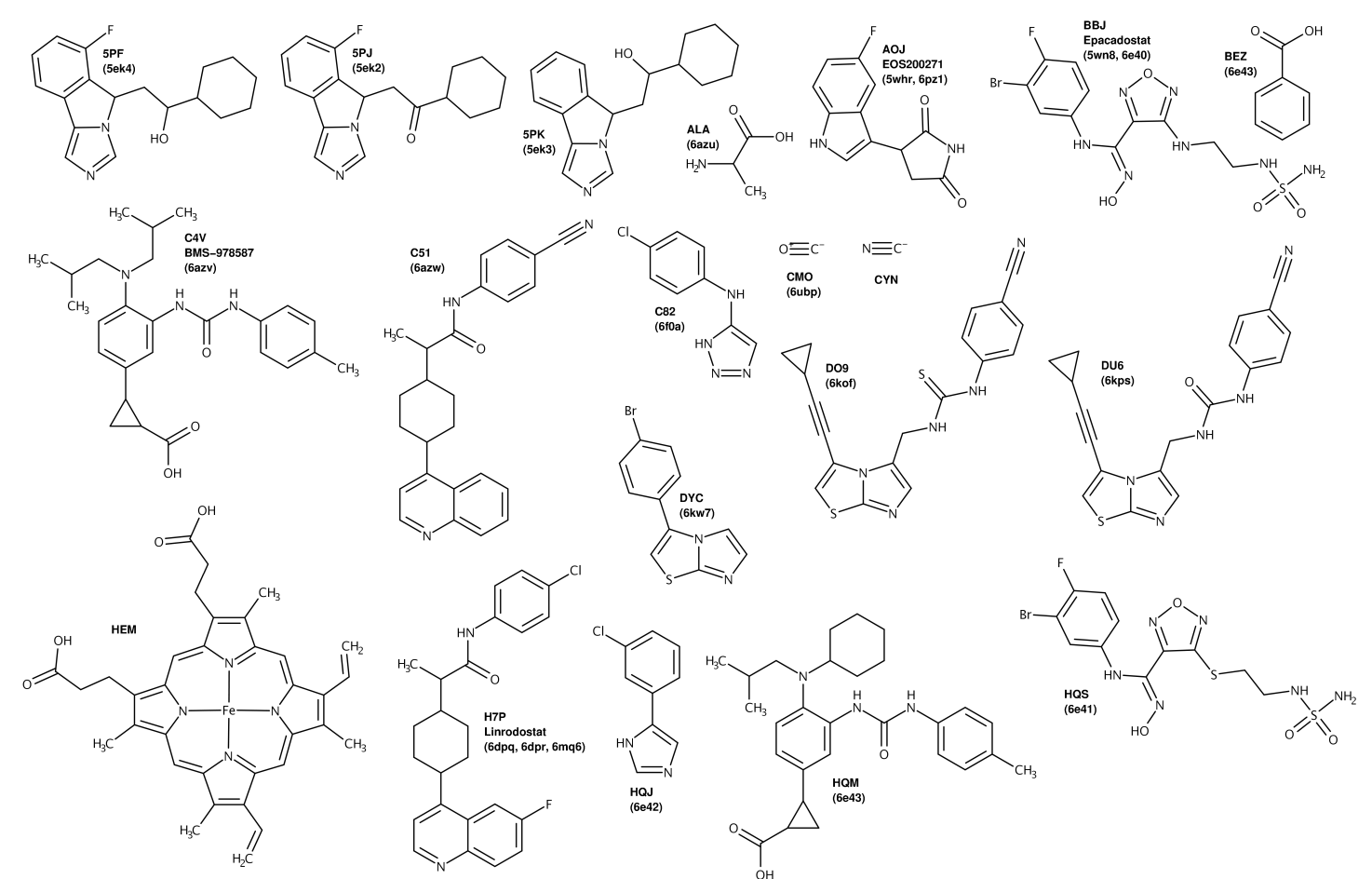

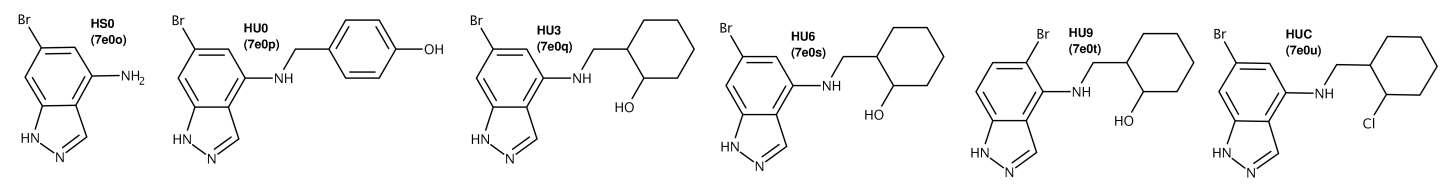

a
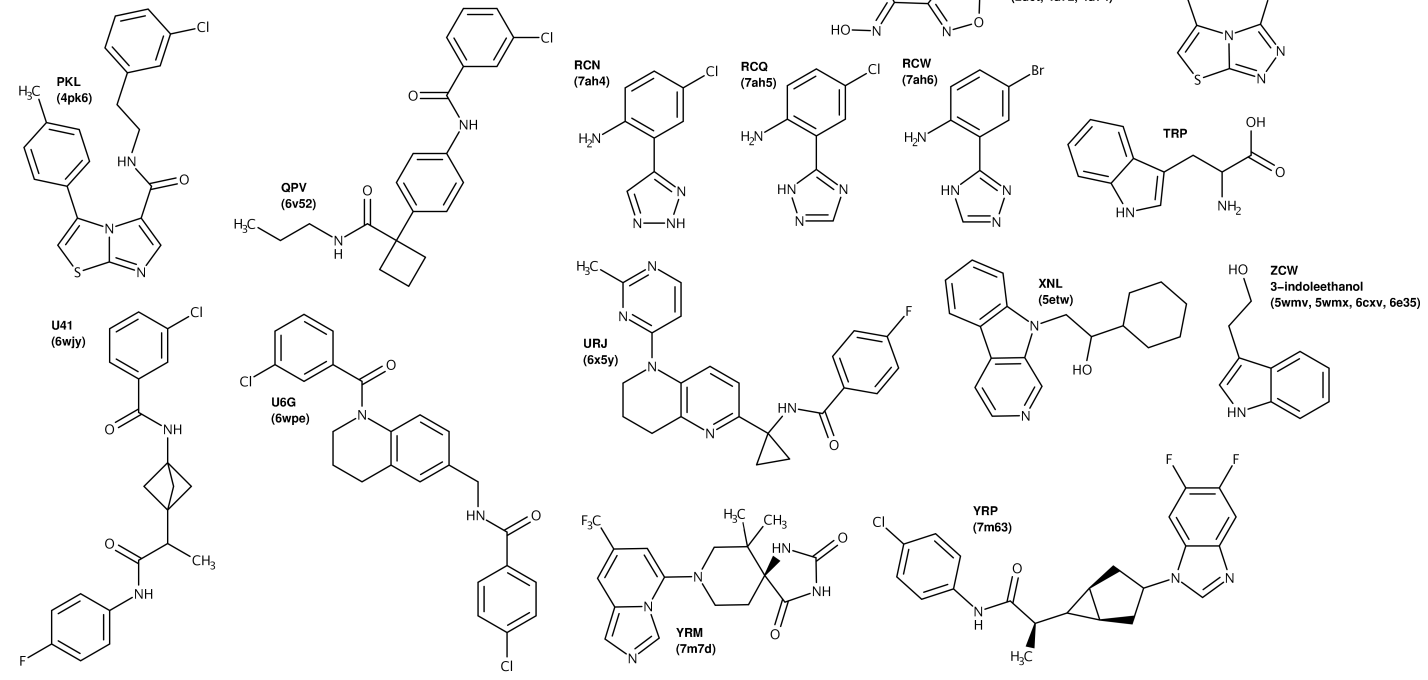

Figure S1: Co-crystallized IDO1 ligands with PDB ligand ID and PDB ID of complex. 
<smiles>CNc1nonc1/C(=N/O)Nc1ccc(F)c(Cl)c1</smiles>
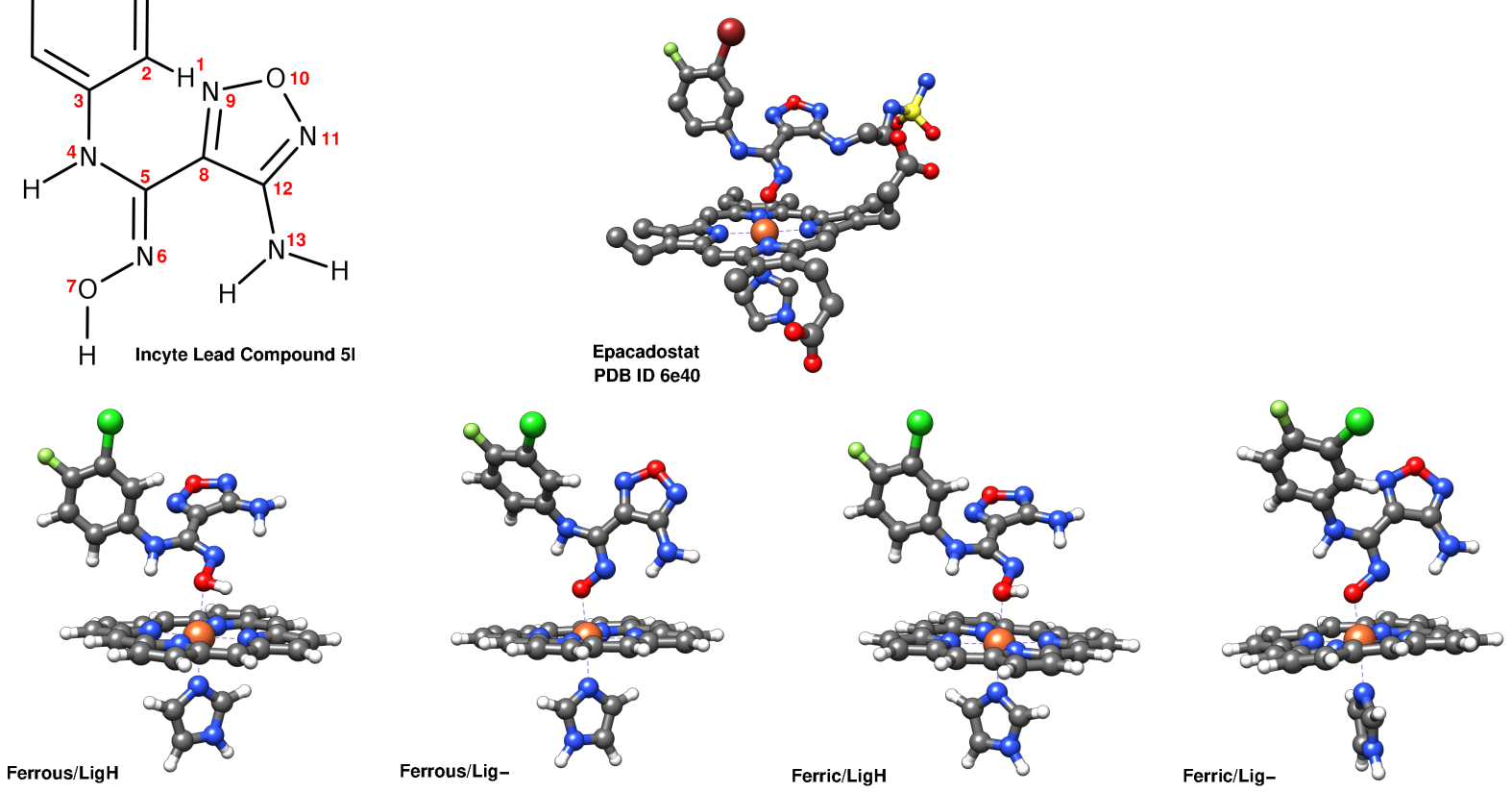

\begin{tabular}{|c|c|c|c|c|c|}
\hline & PDB $6 \mathrm{e} 40$ & $\mathrm{Fe}^{2+} / \operatorname{LigH}$ & $\mathrm{Fe}^{2+} / \mathrm{Lig}^{-}$ & $\mathrm{Fe}^{3+} / \operatorname{LigH}$ & $\mathrm{Fe}^{3+} / \operatorname{Lig}^{-}$ \\
\hline Distance Fe-O7 $[\AA]$ & 1.79 & 2.207 & 1.979 & 2.087 & 1.833 \\
\hline Torsion C2-C3-N4-C5 [ [] & -53 & -34 & 7 & -56 & -20 \\
\hline Torsion C3-N4-C5-N6 [] & 171 & 151 & 136 & 161 & 146 \\
\hline Torsion N4-C5-N6-O7 [ [] & -6 & -3 & -4 & -1 & -4 \\
\hline Torsion N4-C5-C8-N9 [ [ $]$ & -18 & -14 & -3 & -22 & -13 \\
\hline Torsion C5-N6-O7-Fe [] & 122 & 101 & 177 & 113 & 175 \\
\hline Angle Porphine Phenyl [ [] & 88 & 84 & 52 & 81 & 57 \\
\hline Angle Porphine Amidine $\left[^{\circ}\right]$ & 47 & 39 & 85 & 40 & 84 \\
\hline Angle Porphine Furazan [ $\left[^{\circ}\right]$ & 32 & 33 & 74 & 31 & 69 \\
\hline
\end{tabular}

Table S5: Comparison of structural parameters of experimentally determined heme-bound conformations of epacadostat (4, PDB ID 6e40) ${ }^{10}$ and Incyte lead compound 5l (5) from DFT calculations. The structural parameters of the $\mathrm{Fe}^{3+} / \mathrm{LigH}$ complex correspond best to the experimental data. Geometry optimizations were carried out with the PBE0 hybrid functional using the Gaussian09 code and the TZVP basis set, assuming a low-spin complex. 

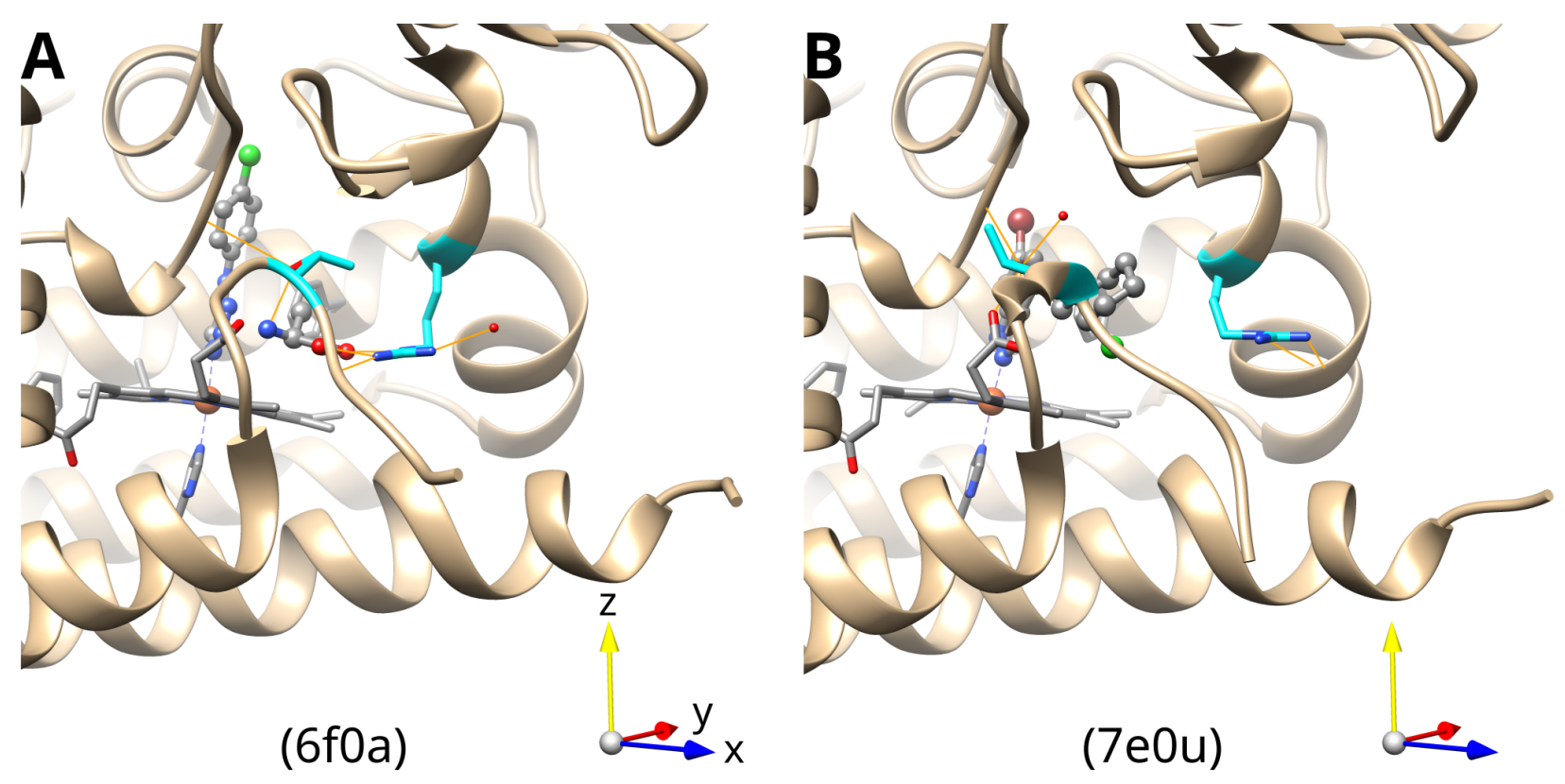

Figure S2: Additional closed JK-loop ${ }^{\mathrm{C}}$ conformations. Residues Thr379 and Arg231 are highlighted in cyan, and their hydrogen bonding network is shown by thin green lines. A. $\mathrm{X}$-ray structure 6f0a. B. X-ray structure $7 \mathrm{e} 0 \mathrm{u}$. 


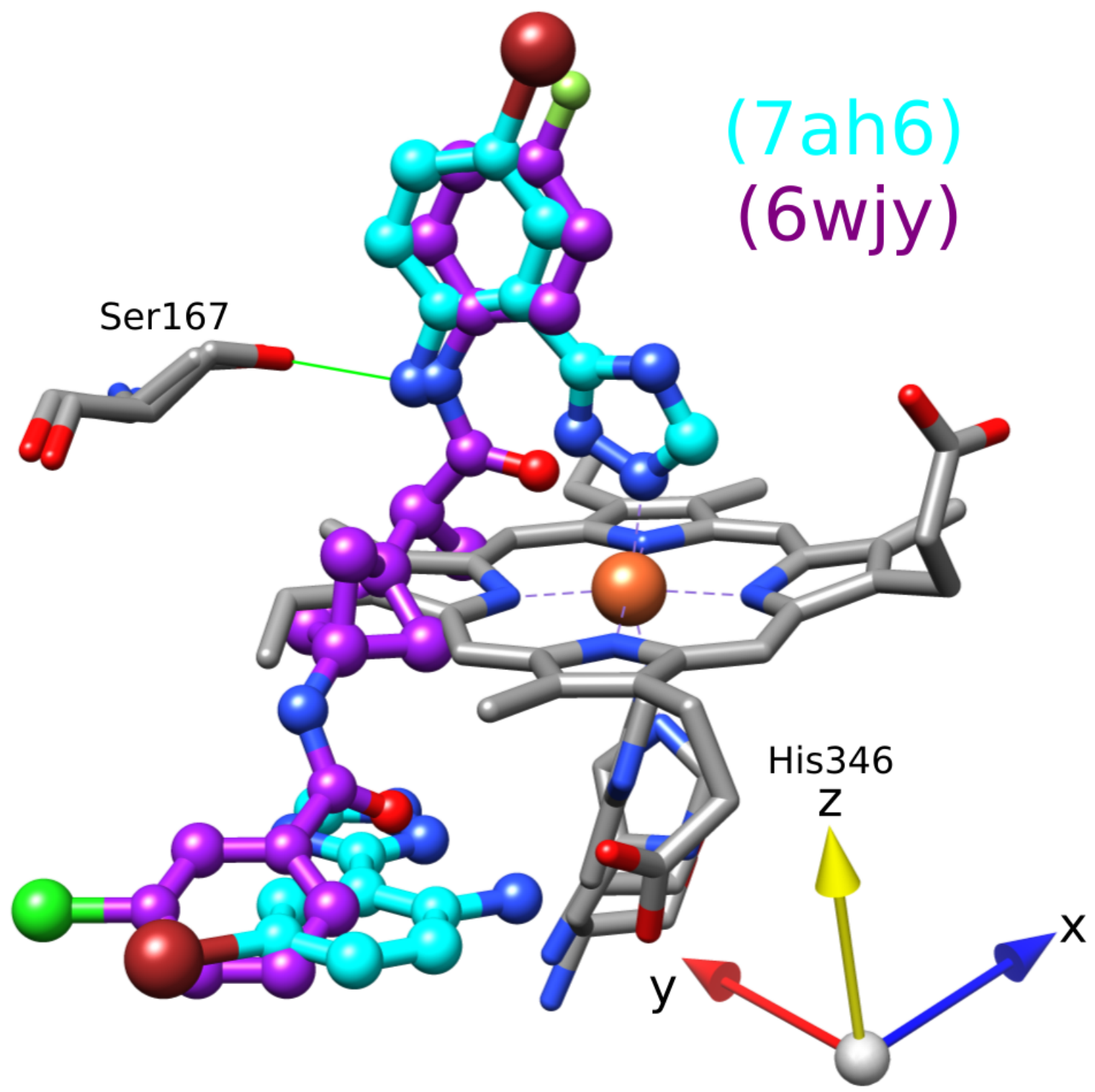

Figure S3: Superposition of holo-IDO1 inhibitor MMG-0752 (cyan) with apo-IDO1 inhibitor from structure 6wjy (purple). The hydrogen bonds with Ser167 are shown as thin green lines. 


\section{ESP calculation}

Electrostatic potentials were calculated with the UHBD code ${ }^{27}$ on the structure $6 \mathrm{e} 46 .{ }^{10}$ The protein was described with the CHARMM27 force field. ${ }^{28,29}$

\section{References}

(1) Sugimoto, H.; Oda, S.-I.; Otsuki, T.; Hino, T.; Yoshida, T.; Shiro, Y. Crystal structure of human indoleamine 2,3-dioxygenase: catalytic mechanism of $\mathrm{O}_{2}$ incorporation by a heme-containing dioxygenase. Proc. Natl. Acad. Sci. U. S. A. 2006, 103, 2611-2616.

(2) Tojo, S.; Kohno, T.; Tanaka, T.; Kamioka, S.; Ota, Y.; Ishii, T.; Kamimoto, K.; Asano, S.; Isobe, Y. Crystal structures and structure-activity relationships of imidazothiazole derivatives as IDO1 inhibitors. ACS Med. Chem. Lett. 2014, 5, 1119-1123.

(3) Peng, Y.-H.; Ueng, S.-H.; Tseng, C.-T.; Hung, M.-S.; Song, J.-S.; Wu, J.-S.; Liao, F.Y.; Fan, Y.-S.; Wu, M.-H.; Hsiao, W.-C.; Hsueh, C.-C.; Lin, S.-Y.; Cheng, C.-Y.; Tu, C.-H.; Lee, L.-C.; Cheng, M.-F.; Shia, K.-S.; Shih, C.; Wu, S. Important hydrogen bond networks in indoleamine 2,3-dioxygenase 1 (IDO1) inhibitor design revealed by crystal structures of imidazoleisoindole derivatives with IDO1. J. Med. Chem. 2016, $59,282-293$.

(4) Crosignani, S.; Bingham, P.; Bottemanne, P.; Cannelle, H.; Cauwenberghs, S.; Cordonnier, M.; Dalvie, D.; Deroose, F.; Feng, J. L.; Gomes, B.; Greasley, S.; Kaiser, S. E.; Kraus, M.; Négrerie, M.; Maegley, K.; Miller, N.; Murray, B. W.; Schneider, M.; Soloweij, J.; Stewart, A. E.; Tumang, J.; Torti, V. R.; Van Den Eynde, B.; Wythes, M. Discovery of a novel and selective indoleamine 2,3-dioxygenase (IDO-1) inhibitor 3(5-fluoro-1H-indol-3-yl)pyrrolidine-2,5-dione (EOS200271/PF-06840003) and its characterization as a potential clinical candidate. J. Med. Chem. 2017, 60, 9617-9629. 
(5) Lewis-Ballester, A.; Pham, K. N.; Batabyal, D.; Karkashon, S.; Bonanno, J. B.; Poulos, T. L.; Yeh, S.-R. Structural insights into substrate and inhibitor binding sites in human indoleamine 2,3-dioxygenase 1. Nat. Commun. 2017, 8, 1693.

(6) Wu, Y.; Xu, T.; Liu, J.; Ding, K.; Xu, J. Structural insights into the binding mechanism of IDO1 with hydroxylamidine based inhibitor INCB14943. Biochem. Biophys. Res. Commun. 2017, 487, 339-343.

(7) Nelp, M. T.; Kates, P. A.; Hunt, J. T.; Newitt, J. A.; Balog, A.; Maley, D.; Zhu, X.; Abell, L.; Allentoff, A.; Borzilleri, R.; Lewis, H. A.; Lin, Z.; Seitz, S. P.; Yan, C.; Groves, J. T. Immune-modulating enzyme indoleamine 2,3-dioxygenase is effectively inhibited by targeting its apo-form. Proc. Natl. Acad. Sci. U. S. A. 2018, 115, 32493254.

(8) Lewis-Ballester, A.; Karkashon, S.; Batabyal, D.; Poulos, T. L.; Yeh, S.-R. Inhibition mechanisms of human indoleamine 2,3 dioxygenase 1. J. Am. Chem. Soc. 2018, 140, 8518-8525.

(9) Pham, K. N.; Yeh, S.-R. Mapping the binding trajectory of a suicide inhibitor in human indoleamine 2,3-dioxygenase 1. J. Am. Chem. Soc. 2018, 140, 14538-14541.

(10) Luo, S.; Xu, K.; Xiang, S.; Chen, J.; Chen, C.; Guo, C.; Tong, Y.; Tong, L. Highresolution structures of inhibitor complexes of human indoleamine 2,3-dioxygenase 1 in a new crystal form. Acta Crystallogr. Sect. F Struct. Biol. Commun. 2018, 74, 717-724.

(11) Alexandre, J. A. C.; Swan, M. K.; Latchem, M. J.; Boyall, D.; Pollard, J. R.; Hughes, S. W.; Westcott, J. New 4-amino-1,2,3-triazole inhibitors of indoleamine 2,3dioxygenase form a long-lived complex with the enzyme and display exquisite cellular potency. ChemBioChem 2018, 19, 552-561.

(12) Peng, Y.-H.; Liao, F.-Y.; Tseng, C.-T.; Kuppusamy, R.; Li, A.-S.; Chen, C.-H.; Fan, Y.S.; Wang, S.-Y.; Wu, M.-H.; Hsueh, C.-C.; Chang, J.-Y.; Lee, L.-C.; Shih, C.; Shia, K.- 
S.; Yeh, T.-K.; Hung, M.-S.; Kuo, C.-C.; Song, J.-S.; Wu, S.-Y.; Ueng, S.-H. Unique Sulfur-Aromatic Interactions Contribute to the Binding of Potent Imidazothiazole Indoleamine 2,3-Dioxygenase Inhibitors. J. Med. Chem. 2020, 63, 1642-1659.

(13) Kumar, S.; Waldo, J. P.; Jaipuri, F. A.; Marcinowicz, A.; Van Allen, C.; Adams, J.; Kesharwani, T.; Zhang, X.; Metz, R.; Oh, A. J.; Harris, S. F.; Mautino, M. R. Discovery of clinical candidate (1R,4R)-4-((R)-2-((S)-6-fluoro-5H-imidazo[5,1-a]isoindol-5yl)-1-hydroxyethyl)cyclohexan-1-ol (navoximod), a potent and selective inhibitor of indoleamine 2,3-dioxygenase 1. J. Med. Chem. 2019, 62, 6705-6733.

(14) Zhang, H.; Liu, K.; Pu, Q.; Achab, A.; Ardolino, M. J.; Cheng, M.; Deng, Y.; Doty, A. C.; Ferguson, H.; Fradera, X.; Knemeyer, I.; Kurukulasuriya, R.; Lam, Y. H.; Lesburg, C. A.; Martinot, T. A.; McGowan, M. A.; Miller, J. R.; Otte, K.; Biju, P. J.; Sciammetta, N.; Solban, N.; Yu, W.; Zhou, H.; Wang, X.; Bennett, D. J.; Han, Y. Discovery of amino-cyclobutarene-derived indoleamine-2,3-dioxygenase 1 (IDO1) inhibitors for cancer immunotherapy. ACS Med. Chem. Lett. 2019, 10, 1530-1536.

(15) Pham, K. N.; Lewis-Ballester, A.; Yeh, S.-r. Structural basis of inhibitor selectivity in human indoleamine 2,3-dioxygenase 1 and tryptophan dioxygenase. J. Am. Chem. Soc. 2019, 141, 18771-18779.

(16) Röhrig, U. F.; Reynaud, A.; Majjigapu, S. R.; Vogel, P.; Pojer, F.; Zoete, V. Inhibition Mechanisms of Indoleamine 2,3-Dioxygenase 1 (IDO1). J. Med. Chem. 2019, 62, 87848795.

(17) Pham, K. N.; Lewis-Ballester, A.; Yeh, S.-R. Conformational plasticity in human hemebased dioxygenases. J. Am. Chem. Soc. 2021, 143, 1836-1845.

(18) White, C.; McGowan, M. A.; Zhou, H.; Sciammetta, N.; Fradera, X.; Lim, J.; Joshi, E. M.; Andrews, C.; Nickbarg, E. B.; Cowley, P.; Trewick, S.; Augustin, M.; von Köenig, K.; Lesburg, C. A.; Otte, K.; Knemeyer, I.; Woo, H.; Yu, W.; Cheng, M.; 
Spacciapoli, P.; Geda, P.; Song, X.; Smotrov, N.; Curran, P.; Heo, M. R.; Abeywickrema, P.; Miller, J. R.; Bennett, D. J.; Han, Y. Strategic incorporation of polarity in heme-displacing inhibitors of indoleamine-2,3-dioxygenase-1 (IDO1). ACS Med. Chem. Lett. 2020, 11, 550-557.

(19) Pu, Q.; Zhang, H.; Guo, L.; Cheng, M.; Doty, A. C.; Ferguson, H.; Fradera, X.; Lesburg, C. A.; McGowan, M. A.; Miller, J. R.; Geda, P.; Song, X.; Otte, K.; Sciammetta, N.; Solban, N.; Yu, W.; Sloman, D. L.; Zhou, H.; Lammens, A.; Neumann, L.; Bennett, D. J.; Pasternak, A.; Han, Y. Discovery of potent and orally available bicyclo[1.1.1]pentane-derived indoleamine-2,3-dioxygenase 1 (IDO1) inhibitors. ACS Med. Chem. Lett. 2020, 11, 1548-1554.

(20) Li, D.; Deng, Y.; Achab, A.; Bharathan, I.; Hopkins, B. A.; Yu, W.; Zhang, H.; Sanyal, S.; Pu, Q.; Zhou, H.; Liu, K.; Lim, J.; Fradera, X.; Lesburg, C. A.; Lammens, A.; Martinot, T. A.; Cohen, R. D.; Doty, A. C.; Ferguson, H.; Nickbarg, E. B.; Cheng, M.; Spacciapoli, P.; Geda, P.; Song, X.; Smotrov, N.; Abeywickrema, P.; Andrews, C.; Chamberlin, C.; Mabrouk, O.; Curran, P.; Richards, M.; Saradjian, P.; Miller, J. R.; Knemeyer, I.; Otte, K. M.; Vincent, S.; Sciammetta, N.; Pasternak, A.; Bennett, D. J.; Han, Y. Carbamate and N-pyrimidine mitigate amide hydrolysis: structure-based drug design of tetrahydroquinoline IDO1 inhibitors. ACS Med. Chem. Lett. 2021, 12, 389396.

(21) Hopkins, B.; Zhang, H.; Bharathan, I.; Li, D.; Pu, Q.; Zhou, H.; Martinot, T. A.; Fradera, X.; Lammens, A.; Lesburg, C. A.; Cohen, R. D.; Ballard, J.; Knemeyer, I.; Otte, K.; Vincent, S.; Miller, J. R.; Solban, N.; Cheng, M.; Geda, P.; Smotrov, N.; Song, X.; Bennett, D. J.; Han, Y. Utilization of Metabolite Identification and Structural Data to Guide Design of Low-Dose IDO1 Inhibitors. ACS Med. Chem. Lett. 2021, 12, 1435-1440.

(22) Mirgaux, M.; Leherte, L.; Wouters, J. Influence of the presence of the heme cofactor 
on the JK-loop structure in indoleamine 2,3-dioxygenase 1. Acta Crystallogr. Sect. D Struct. Biol. 2020, 76, 1211-1221.

(23) Röhrig, U. F.; Majjigapu, S. R.; Reynaud, A.; Pojer, F.; Dilek, N.; Reichenbach, P.; Ascencao, K.; Irving, M.; Coukos, G.; Vogel, P.; Michielin, O.; Zoete, V. Azole-based indoleamine 2,3-dioxygenase 1 (IDO1) inhibitors. J. Med. Chem. 2021, 64, 2205-2227.

(24) Ning, X.-L.; Li, Y.-Z.; Huo, C.; Deng, J.; Gao, C.; Zhu, K.-R.; Wang, M.; Wu, Y.X.; Yu, J.-L.; Ren, Y.-L.; Luo, Z.-Y.; Li, G.; Chen, Y.; Wang, S.-Y.; Peng, C.; Yang, L.-L.; Wang, Z.-Y.; Wu, Y.; Qian, S.; Li, G.-B. X-ray structure-guided discovery of a potent, orally bioavailable, dual human indoleamine/tryptophan 2,3-dioxygenase (hIDO/hTDO) inhibitor that shows activity in a mouse model of parkinson's disease. J. Med. Chem. 2021, 64, 8303-8332.

(25) Hamilton, M. M.; Mseeh, F.; McAfoos, T. J.; Leonard, P. G.; Reyna, N. J.; Harris, A. L.; Xu, A.; Han, M.; Soth, M. J.; Czako, B.; Theroff, J. P.; Mandal, P. K.; Burke, J. P.; Virgin-Downey, B.; Petrocchi, A.; Pfaffinger, D.; Rogers, N. E.; Parker, C. A.; Yu, S. S.; Jiang, Y.; Krapp, S.; Lammens, A.; Trevitt, G.; Tremblay, M. R.; Mikule, K.; Wilcoxen, K.; Cross, J. B.; Jones, P.; Marszalek, J. R.; Lewis, R. T. Discovery of IACS-9779 and IACS-70465 as potent inhibitors targeting indoleamine 2,3-dioxygenase 1 (IDO1) apoenzyme. J. Med. Chem. 2021, 64, 11302-11329.

(26) Cereto-Massagué, A.; Ojeda, M. J.; Joosten, R. P.; Valls, C.; Mulero, M.; Salvado, M. J.; Arola-Arnal, A.; Arola, L.; Garcia-Vallvé, S.; Pujadas, G. The good, the bad and the dubious: VHELIBS, a validation helper for ligands and binding sites. J. Cheminform. 2013, 5, 1-9.

(27) Madura, J. D.; Briggs, J. M.; Wade, R. C.; Davis, M. E.; Luty, B. a.; Ilin, A.; Antosiewicz, J.; Gilson, M. K.; Bagheri, B.; Scott, L.; McCammon, J. Electrostatics and 
diffusion of molecules in solution: simulations with the University of Houston Brownian Dynamics program. Comput. Phys. Commun. 1995, 91, 57-95.

(28) MacKerell, A. D.; Bashford, D.; Bellott, M.; Dunbrack, R. L.; Evanseck, J. D.; Field, M. J.; Fischer, S.; Gao, J.; Guo, H.; Ha, S.; Joseph-McCarthy, D.; Kuchnir, L.; Kuczera, K.; Lau, F. T. K.; Mattos, C.; Michnick, S.; Ngo, T.; Nguyen, D. T.; Prodhom, B.; Reiher, W. E.; Roux, B.; Schlenkrich, M.; Smith, J. C.; Stote, R.; Straub, J.; Watanabe, M.; Wiórkiewicz-Kuczera, J.; Yin, D.; Karplus, M. All-Atom Empirical Potential for Molecular Modeling and Dynamics Studies of Proteins. J. Phys. Chem. B 1998, 102, 3586-3616.

(29) MacKerell, A. D. Empirical force fields for biological macromolecules: Overview and issues. J. Comput. Chem. 2004, 25, 1584-1604. 